\title{
Electron Dynamics in AC-Driven Quantum Dots
}

\author{
C.E. Creffield ${ }^{1,2}$ and G. Platero ${ }^{1}$ \\ 1. Instituto de Ciencia de Materiales de Madrid (CSIC), Cantoblanco, E-28049, \\ Madrid, Spain \\ 2 Dipartimento di Fisica, Università di Roma "La Sapienza", Piazzale Aldo Moro \\ 2, I-00185 Roma, Italy
}

Summary. We investigate the dynamics of interacting electrons confined to two types of quantum dot system, when driven by an external AC field. We first consider a system of two electrons confined to a pair of coupled quantum dots [1] by using an effective two-site model of Hubbard-type. Numerically integrating the Schrödinger equation in time reveals that for certain values of the strength and frequency of the field the tunneling between the dots can be destroyed, thus allowing the correlated two-electron states to be manipulated. We then show how Floquet theory 2 can be used to predict the field parameters at which this effect occurs.

We then consider the case of confining the electrons to a single two-dimensional quantum dot in the limit of low particle-density. In this system the electrons form strongly correlated states termed Wigner molecules, in which the Coulomb interaction causes them to become highly localised in space. Again using an effective model of Hubbard-type, we investigate how the AC field can drive the dynamics of the Wigner states [3]. As before, we find that the AC field can be used to control the tunneling between various charge configurations, and we relate this to the presence of avoided crossings in the Floquet quasi-energy spectrum. These results hold out the exciting possibility of using AC fields to control the time evolution of entangled states in mesoscopic devices, which has great relevance to the rapidly advancing field of quantum information processing. 


\section{Introduction}

A quantum dot (QD) is a structure in which electrons can be confined to small length scales, comparable to their Fermi wavelength. A set of electrons held in such a structure is conceptually similar to a set of atomic electrons bound to a nucleus, and for this reason quantum dots are sometimes termed "artificial atoms" [4. Unlike real atoms, the physical properties of quantum dots can be easily varied, which gives theorists and experimentalists the opportunity to study novel quantum effects in a well-controlled system.

To extend the atomic analogy further, we can consider linking QDs together to form "artificial molecules". By allowing electrons to tunnel between the QDs, the electronic states on the QDs can hybridize, and form new states that extend over the whole system 5 . The degree of the tunneling determines the strength of this hybridization. If the tunneling is weak the electrons remain essentially localised on the QDs in analogy with ionic bonding states. Conversely, if the tunneling is strong then the electrons form delocalised states with a covalent character. Recent transport experiments using AC potentials [6] have been performed on double QD systems, and have indeed revealed the ionic or covalent character of the electronic states by measurement of the induced photo-current.

Ever since the pioneering work of Anderson [7], it has been known that random spatial disorder can cause electronic states to become localised in quantum systems. More recently it has been found that an AC driving field can produce a similar intriguing effect termed dynamical localisation, in which the tunneling dynamics of a particle can be destroyed. One of the first systems in which this effect was predicted is that of a particle moving in a doublewell potential [8]. A physical realization of this could consist of two coupled QDs containing a single electron - the simplest type of artificial molecule possible. If this system is prepared with the electron occupying one of the QDs, we can expect it to tunnel across to the other QD on a time scale set by the Rabi frequency. However, if an AC field of the correct strength and frequency is applied to the system, the tunneling is destroyed, and the particle will remain trapped in the initial well.

Weak time-dependent fields are generally treated as small perturbations, which produce transitions between the eigenstates of the unperturbed quantum system. This approach, however, is not applicable to treat the strong driving fields required to produce dynamical localisation, and instead the technique of Floquet analysis [2], which is valid in all regimes of driving, has proven to be extremely effective. In this approach, which we briefly outline in the next section, the important quantities to calculate are the quasi-energies, which play a similar role in driven systems to the eigenenergies in the undriven case. In particular, dynamical localisation occurs when two quasi-energies of states participating in the dynamics approach each other, and become either degenerate (a crossing) or close to degenerate (an avoided crossing). Using this formalism, analytic and numerical studies of the double-well system have 
shown 19,10,11] that in the limit of high frequencies, quasi-energy crossings occur when the ratio of the field strength to the frequency is a root of the Bessel function $J_{0}$.

Adding a second electron to the coupled QD system, however, introduces considerable complications. At the low electron densities typically present in QDs, strong correlations produced by the Coulomb interaction can significantly influence the electronic structure. One of the most dramatic consequences of this is the formation of Wigner molecule states 12. Understanding the interplay between electron correlations and the driving field is, however, extremely desirable, as the ability to rapidly control electrons using AC fields [13 has immediate applications to quantum metrology 14] and quantum information processing. In particular, manipulating entangled electrons on short timescales is of great importance to the field of quantum computation [15.

We study this problem here by applying the Floquet formalism to systems of interacting particles. The first system we consider is that of two interacting electrons confined to a pair of coupled QDs. A consequence of the interaction is that the system only responds strongly to the field when the field frequency is in resonance with the Coulomb interaction energy. When this condition is satisfied we find that, as for the single-particle case, coherent destruction of tunneling (CDT) can again occur, but that it governed by the roots of the higher-order Bessel functions. We then go on to consider a two-dimensional QD in the Wigner regime, which may also be described by a lattice model of Hubbard-type [16]. Using the same approach we show that CDT can again occur when similar conditions are satisfied, and we clarify how an applied AC field can drive charge redistributions within a strongly correlated QD. Finally we summarize our results and give some brief conclusions.

\section{Methods and approaches}

\subsection{Introduction to Floquet theory}

We consider a general quantum system driven by a periodic electric field, described by a time-dependent Hamiltonian which we can divide in the following way:

$$
H(t)=H_{t}+H_{I}+H_{A C}(t), \quad H_{A C}(t)=H_{A C}(t+n T) .
$$

Here $H_{t}$ holds the tunneling terms, $H_{I}$ holds the electron-electron interaction terms and $H_{A C}(t)$ describes the interaction of the system with the $T$-periodic driving field. The periodicity of the driving field allows us to use the Floquet theorem to write solutions of the Schrödinger equation as $\psi(t)=\exp \left[-i \epsilon_{j} t\right] \phi_{j}(t)$ where $\epsilon_{j}$ is called the quasi-energy, and $\phi_{j}(t)$ is a function with the same period as the driving field, called the Floquet state. This type of expression is familiar in the context of solid-state physics, where 
spatial periodicity permits an analogous rewriting of the spatial wavefunction in terms of quasi-momenta and Bloch states (Bloch's theorem).

The Floquet states provide a complete basis, and thus the time-evolution of a general state may be written as:

$$
|\Psi(t)\rangle=\sum_{j}\left(c_{j} \mathrm{e}^{-i \epsilon_{j} t}\right)\left|\phi_{j}(t)\right\rangle,
$$

which is formally analogous to the standard expansion in the eigenvectors of a time-independent Hamiltonian. Indeed, in the adiabatic limit, $T=2 \pi / \omega \rightarrow$ $\infty$, the quasi-energies evolve to the eigenenergies, and the Floquet states to the eigenstates. It is important to note that in this expansion both the basis vectors (the Floquet states) and the expansion coefficients explicitly depend on time. The nature of this time-dependence is very different however, and the superposition of the $T$-periodicity of the Floquet states with the phase factors arising from the quasi-energies produces a highly complicated, quasiperiodic time-dependence in general. As the Floquet states have the same period as the driving field, they are only able to produce structure in the time-dependence on short time-scales. Consequently, the dynamics of the system on time-scales much larger than $T$ is essentially determined by just the quasi-energies, and hence evaluating the quasi-energies provides a simple and direct way of investigating this behavior. In particular, when two quasienergies approach degeneracy the time-scale for tunneling between the states diverges, producing the phenomenon of CDT.

As we shall see for the specific quantum systems we consider, it is frequently the case that the total Hamiltonian is invariant under the generalized parity operation: $x \rightarrow-x ; t \rightarrow t+T / 2$. As a result the Floquet states can also be classified into parity classes, depending whether they are odd or even under this parity operation. Quasi-energies belonging to different parity classes may cross as an external parameter (such as the field strength) is varied, but if they belong to the same class the von Neumann-Wigner [17] theorem forbids this, and the closest approaches possible are avoided crossings. Identifying the presence of crossings and avoided crossings in the quasi-energy spectrum thus provides a necessary (though not sufficient) condition for CDT to occur.

\subsection{Perturbation theory for Floquet states}

Although the quasi-energies are extremely useful for interpretation of the time-dependence of a quantum system, they are usually difficult to calculate and numerical methods must be employed. When the driving field dominates the dynamics, however, it is possible to use a form of perturbation theory introduced by Holthaus [10], in which the time-dependent part of the problem is solved exactly, and tunneling part of the Hamiltonian, $H_{t}$, acts as the perturbation. This was generalized to treat interacting systems in Refs. [1, 3 and was found to be very successful in the high-frequency regime, where $\hbar \omega$ is the dominant energy-scale. We now give a brief outline of this method. 
The Floquet states and their quasi-energies may be conveniently obtained from the eigenvalue equation:

$$
\left(H(t)-i \hbar \frac{\partial}{\partial t}\right)\left|\phi_{j}(t)\right\rangle=\epsilon_{j}\left|\phi_{j}(t)\right\rangle
$$

where we consider the operator $[H(t)-i \hbar \partial / \partial t]$ to operate in an extended Hilbert space of $T$-periodic functions [18]. The procedure consists of dividing the Hamiltonian as in Eq.11, and finding the eigensystem of the operator $\left[H_{I}+H_{A C}(t)-i \hbar \partial / \partial t\right]$, while regarding the tunneling Hamiltonian $H_{t}$ as acting as a perturbation. Standard Rayleigh-Schrödinger perturbation theory can now be used to evaluate the order-by-order corrections to this result, requiring only that we define an appropriate inner product for the extended Hilbert space:

$$
\left\langle\left\langle\phi_{m} \mid \phi_{n}\right\rangle\right\rangle_{T}=\frac{1}{T} \int_{0}^{T}\left\langle\phi_{m}\left(t^{\prime}\right) \mid \phi_{n}\left(t^{\prime}\right)\right\rangle d t^{\prime} .
$$

Here $\langle\cdot \mid \cdot\rangle$ denotes the usual scalar product for the spatial component of the wavevectors, and $\langle\cdot \mid \cdot\rangle_{T}$ is the integration over the compact time coordinate. We shall show in later sections how this method can be used to obtain analytical forms which accurately describe the behavior of the quasi-energies for the systems we study.

\subsection{Numerical methods}

To study the time-evolution of each system, we used a fourth-order RungeKutta method to evolve a given initial state in time - typically of the order of fifty periods of the driving field. Throughout the time-evolution, physical quantities such as the number occupation of a given site were measured, and it was ensured that the unitarity of the wavefunction was accurately preserved.

A number of different methods can be used to numerically calculate the quasi-energies of a quantum system, and a detailed description of them is given in Ref.[2]. One technique well-suited to our approach is to evaluate the unitary time-evolution operator for one period of the driving field $U(t+T, t)$, and then to diagonalize it. It may be easily shown that the eigenvectors of this operator are equal to the Floquet states, and its eigenvalues are related to the quasi-energies via $\lambda_{j}=\exp \left[-i \epsilon_{j} T\right]$. This method is particularly convenient for our purposes, as $U(T, 0)$ can be obtained by propagating the unit matrix in time over one period of the field, using the same Runge-Kutta method described above.

\section{The driven double quantum-dot}

We consider a simplified model of this system, in which each QD is replaced by a single site. Electrons are able to tunnel between the sites, and we include 
the effect of interactions by means of a Hubbard- $U$ term:

$$
H=\tilde{t} \sum_{\sigma}\left(c_{1 \sigma}^{\dagger} c_{2 \sigma}+\text { H.c. }\right)+\sum_{i=1}^{2}\left(\tilde{U} n_{i \uparrow} n_{i \downarrow}+E_{i}(t) n_{i}\right) .
$$

Here $\tilde{t}$ is the hopping parameter, and for the remainder of this work we shall take $\hbar$ equal to one, and measure all energies in units of $\tilde{t} . E_{i}(t)$ is the external electric potential applied to site $i$. Clearly only the potential difference, $E_{1}-E_{2}$, is of physical importance, so we may choose to take the symmetric parametrization:

$$
E_{1}(t)=\frac{E}{2} \cos \omega t, \quad E_{2}(t)=-\frac{E}{2} \cos \omega t .
$$

The Hilbert space of Hamiltonian (5) is six-dimensional, comprising three singlet states and a three dimensional triplet space. Measurements on semiconductor QDs have shown that the spin-flip relaxation time is typically extremely long [19], and so we have chosen not to include any spin-flip terms in the Hamiltonian. Consequently the singlet and triplet sectors are completely decoupled, and so if the initial state possesses a definite parity this will be retained throughout its time-evolution, and only states of the same parity need to be included in the basis.

To study the time evolution of the system, we used the ground state of the static Hamiltonian (a singlet) as the initial state, and evolved it in time as described in Section 2.3. Three probability functions were measured throughout the time evolution: $p_{L L}(t), p_{R R}(t)$ and $p_{R L}(t)$, which are respectively the probability that both electrons are in the left QD, both are in the right $\mathrm{QD}$, and that one electron is in each of the QDs. The Coulomb interaction favors separating the electrons, and thus for strong interactions the ground-state has a large value of $p_{R L}$, and relatively small values of $p_{L L}$ and $p_{R R}$. We show in Fig. 1 the time evolution of these quantities for $\tilde{U}=8$ and $\omega=4$, at two different values of electric potential. In both cases the detailed form of the time-evolution is highly complicated, but it is clear that the system behaves in two distinct ways. In Fig. 17a the value of $p_{R L}$ periodically cycles between its initial high value (indicating that each dot holds approximately one electron) to nearly zero, while the values of $p_{L L}$ and $p_{R R}$ correspondingly rise and fall at its expense. This behavior is very different to that shown in Fig. 11b, where $p_{R L}$ never drops below a value of 0.78 , and the other two probabilities oscillate with a very small amplitude. It thus appears that CDT is occurring in the second case, and that the system's time evolution is essentially frozen. We shall term the minimum value of $p_{R L}$ attained during the time-evolution $p_{\min }$, and use this to quantify whether CDT occurs, as a high value of $p_{\min }$ signifies that tunneling has been destroyed, while a low value indicates that the electrons are free to move between the QDs.

In Fig.2 $2 \mathrm{~b}$ we present a contour plot of $p_{\min }$ as a function of both of the frequency and strength of the AC field. Dark areas correspond to low values 


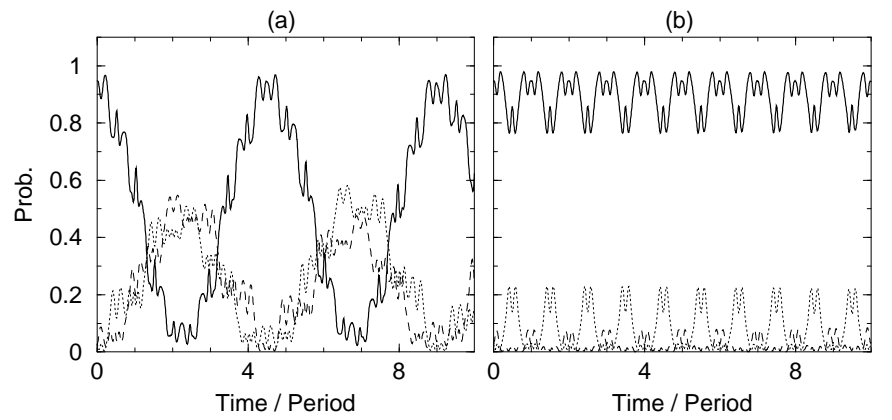

Fig. 1. Time evolution of the driven double QD system for $\tilde{U}=8$ and $\omega=4$. (a) electric potential, $E=30.0$; (b) $E=33.5$. Thick solid line $=p_{R L}(t)$, dotted line $=$ $p_{L L}(t)$, dashed line $=p_{R R}(t)$.

of $p_{\min }$, and it can be seen that they form horizontal bands, indicating that the system is excited strongly by the AC field only at "resonant" values of $\omega$. Close examination of this plot reveals that these bands occur at frequencies $\omega=\tilde{U}, \tilde{U} / 2, \tilde{U} / 3 \ldots$, at which the system can absorb an integer number of photons to overcome the Coulomb repulsion between electrons, thereby enabling tunneling processes such as $|\uparrow, \downarrow\rangle \rightarrow|0, \uparrow \downarrow\rangle$ to occur. We can additionally observe that these bands are punctuated by narrow zones in which CDT occurs. Their form can be seen more clearly in the cross-section of $p_{\text {min }}$ given in Fig.3a, which reveals them to be narrow peaks. These peaks are approximately equally spaced along each resonance, the spacing increasing with $\omega$. In Fig.2a we show another contour plot of $p_{\min }$, this time obtained from a full simulation of detailed physical model of two interacting electrons confined to a pair of coupled GaAs QDs (for more details on this simulation see Ref.[1]). The striking similarity between these results clearly indicates that our simple, effective model (5) indeed captures the essential processes occurring in the full system.

We emphasize that these results are radically different to those obtained for non-interacting particles. In this case an analogous plot of delocalisation shows a fan-like structure [11], in which localisation occurs along lines given by $\omega=E / x_{j}$, where $x_{j}$ is the $j$-th root of the Bessel function $J_{0}(x)$. As a test of our method, we repeated our investigation with the inter-electron Coulomb repulsion $\tilde{U}$ set to zero, and found that the fan structure was indeed reproduced.

In Fig. 3a we show the Floquet quasi-energies as a function of the field strength for $\omega=2$, one of the resonant frequencies visible in Fig.2b. We see that the system possesses two distinct regimes of behavior, depending on whether the driving potential is weaker of stronger than $\tilde{U}$. For weak fields $E<\tilde{U}$, as studied previously in Ref. [20], the Floquet spectrum consists 

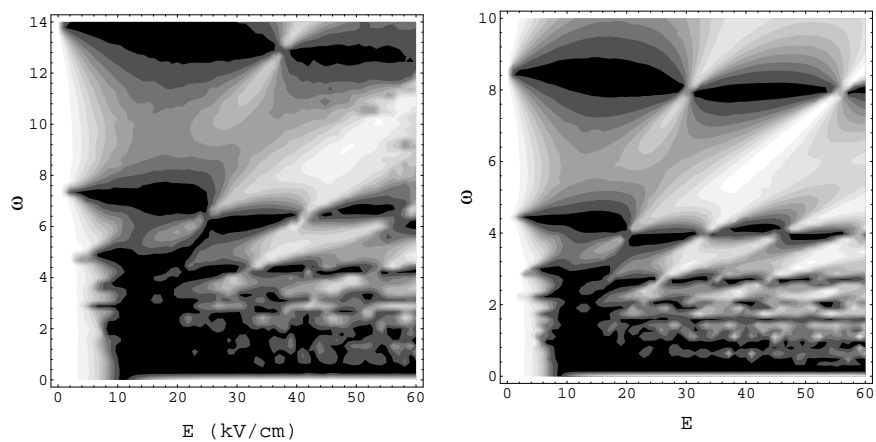

Fig. 2. $p_{\min }$ as a function of the strength $E$ and energy $\hbar \omega$ of the AC field: (a) for a full simulation of a QD system ( $\hbar \omega$ in units of meV) (b) for the two-site model with $\tilde{U}=8$ (both axes in units of $\tilde{t})$.

of one isolated state (which evolves from the ground state) and two states which make a set of exact crossings. Although in this regime $p_{\min }$ shows little structure, these crossings do in fact influence the system's dynamics. To demonstrate this, we show in Fig the Floquet quasi-energies in the weakfield regime for the case of $\tilde{U}=16$, and plot beneath it the minimum value of $p_{L L}$ attained during the time-evolution, where this time the state $|\uparrow \downarrow, 0\rangle$ has been used as the initial state. It can be seen that for this choice of initial condition, the crossings of the quasi-energies again produce CDT and freeze the initial state - despite the Coulomb repulsion between the electrons.

This surprising result may be understood as follows. For large values of $\tilde{U}$, the singlet eigenstates of the undriven system consist of the ground state, separated by the Hubbard gap $\tilde{U}$ from two almost degenerate excited states. For small values of the driving potential, the two excited states remain isolated from the ground state, and constitute an effective two-level system with a level-splitting of $\Delta \simeq 4 \tilde{t}^{2} / \tilde{U}$. Thus if the system is prepared in an initial state which projects mainly onto the excited states, its dynamics will be governed by the two-level approximation [9 10,11], and CDT will occur at the roots of $J_{0}$. We show in Fig 4 a the quasi-energies obtained from the twolevel approximation, which give excellent agreement with the actual results with no adjustable parameters. As $E$ becomes comparable to the Hubbard gap, however, the two excited states are no longer isolated from the ground state, and all three levels must be taken into account. This can be seen in the progressive deviation of the quasi-energies from the two-level approximation as the electric potential approaches $\tilde{U}$.

When the electric potential exceeds $\tilde{U}$, the system displays a very different behavior, in which $p_{\min }$ remains close to zero except at a series of narrow peaks, corresponding to the close approaches of two of the quasi-energies. A detailed examination of these approaches (see Fig. $3 \mathrm{~b}$ ) reveals them to be 
avoided crossings between the Floquet states which evolve from the ground state and the higher excited state, and have the same generalized parity. The remaining state, of opposite parity, makes small oscillations around zero, but its exact crossings with the other two states do not correlate with any structure in $p_{\min }$.

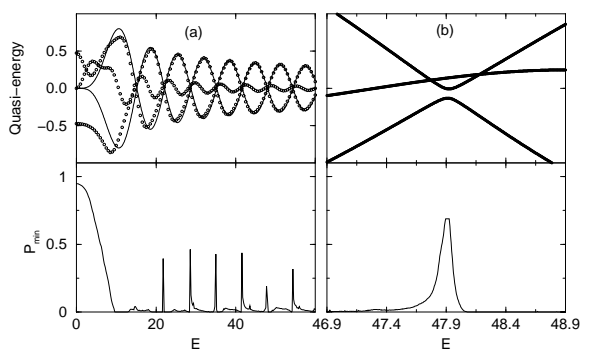

Fig. 3. (a) Quasi-energy spectrum for the two-site model for $\tilde{U}=8$ and $\omega=2$, circles $=$ exact results, lines $=$ perturbation theory, (b) magnified view of exact results for a single avoided crossing. Beneath are the corresponding plots of $p_{\min }$.

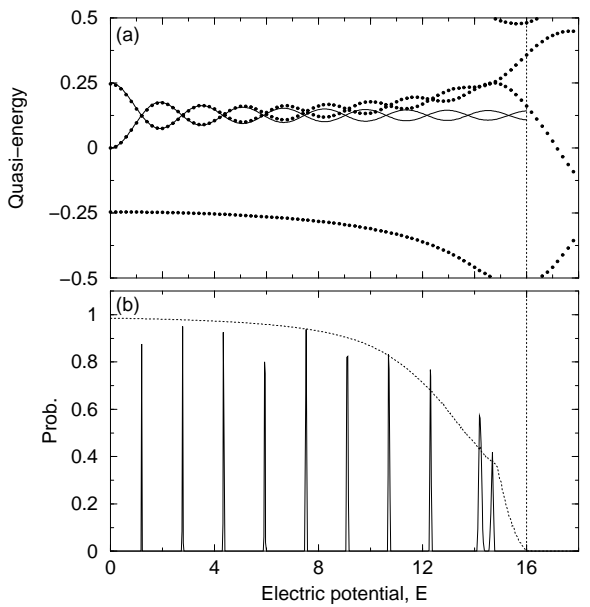

Fig. 4. (a) Quasi-energy spectrum for the two-site model for $\tilde{U}=16$ and $\omega=2$, circles $=$ exact results, solid line $=$ two-level approximation, $\epsilon_{ \pm}= \pm(\Delta / 2) J_{0}(2 E / \omega)$. (b) Solid line $=$ minimum value of $p_{L L}(t)$, starting from the initial configuration $|\uparrow \downarrow, 0\rangle$. The dotted line denotes $p_{\text {min }}$, which shows little structure. In both plots the vertical dotted line marks the transition to the strong-driving regime. 
To interpret this behavior in the strong-field regime, we now obtain analytic expressions for the quasi-energies via the perturbation theory described in Section 2.2. The first step is to solve the eigenvalue equation (3) in the absence of the tunneling component $H_{t}$. In a real-space representation the interaction terms are diagonal, and so it can be readily shown that an orthonormal set of eigenvectors is given by:

$$
\begin{aligned}
\left|\epsilon_{0}(t)\right\rangle & =\left(\exp \left[i \epsilon_{0} t\right], 0,0\right) \\
\left|\epsilon_{+}(t)\right\rangle & =\left(0, \exp \left[-i\left(\tilde{U}-\epsilon_{+}\right) t+i \frac{E}{\omega} \sin \omega t\right], 0\right) \\
\left|\epsilon_{-}(t)\right\rangle & =\left(0,0, \exp \left[-i\left(\tilde{U}-\epsilon_{-}\right) t-i \frac{E}{\omega} \sin \omega t\right]\right)
\end{aligned}
$$

Imposing $T$-periodic boundary conditions reveals the corresponding eigenvalues (modulo $\omega$ ) to be $\epsilon_{0}=0$ and $\epsilon_{ \pm}=\tilde{U}$. These eigenvalues represent the zeroth-order approximation to the Floquet quasi-energies, and for frequencies such that $\tilde{U}=n \omega$ all three eigenvalues are degenerate. This degeneracy is lifted by the perturbation $H_{t}$, and to first-order, the quasi-energies are obtained by diagonalizing the perturbing operator $P_{i j}=\left\langle\left\langle\epsilon_{i}\left|H_{t}\right| \epsilon_{j}\right\rangle\right\rangle_{T}$. By using the well-known identity:

$$
\exp [-i \beta \sin \omega t]=\sum_{m=-\infty}^{\infty} J_{m}(\beta) \exp [-i m \omega t]
$$

to rewrite the form of $\left|\epsilon_{ \pm}(t)\right\rangle$, the matrix elements of $P$ can be obtained straightforwardly, and its eigenvalues subsequently found to be $\epsilon_{0}=0$ and $\epsilon_{ \pm}= \pm 2 J_{n}(E / \omega)$. Fig. Ba demonstrates the excellent agreement between this result (with $n=4$ ) and the exact quasi-energies for strong and moderate fields, which allows the position of the peaks in $p_{\text {min }}$ to be found by locating the roots of $J_{n}$. Similar excellent agreement occurs at the other resonances. For weak fields, however, the interaction terms do not dominate the tunneling terms and the perturbation theory breaks down, although we are still able to treat the system phenomenologically by using the effective two-level approximation.

\section{The square quantum dot}

\subsection{What is a Wigner molecule?}

As we remarked in the Introduction, the Coulomb interaction between the electrons can significantly affect the electronic structure of a QD. Such strongly correlated problems are notoriously difficult to treat, and the addition of a time-dependent field complicates the problem even further. When the mean inter-electron separation exceeds a certain critical value, however, a surprising simplification occurs, as the Coulomb interaction dominates the kinetic 
energy and drives a transition to a quasi-crystalline arrangement which minimizes the total electrostatic energy. In analogy to the phenomenon of Wigner crystallization in bulk two-dimensional systems 21,22] such a state is termed a Wigner molecule [12]. As the electrons in the Wigner state are sharply localised in space, the system can be naturally and efficiently discretized by placing lattice points just at these spatial locations. A many-particle basis can then be constructed by taking Slater determinants of single-particle states defined on these lattice sites, from which an effective Hamiltonian of Hubbard-type can be generated to describe the low-energy dynamics of the system [16]. A major advantage of this technique over standard discretization [23,24] schemes, in which a very large number of lattice points is taken to approximate the continuum limit, is that the dimension of the effective Hamiltonian is much smaller (typically by many orders of magnitude), which permits the investigation of systems which would otherwise be prohibitively complex. This approach has proven to be extremely successful in treating a variety of static problems, including one-dimensional QDs [16], two-dimensional QDs with polygonal boundaries 25,26], and electrons confined to quantum rings [27.28]. We further develop this method here by including a time-dependent electric field, and study the temporal dynamics of the system as it is driven out of equilibrium.

\subsection{Model and Methods}

We consider a system of two electrons confined to a square QD with a hardwall confining potential - a simple representation of a two-dimensional semiconductor QD. Such a system can be produced by gating a two-dimensional electron gas confined at a heterojunction interface, and by placing a gate split into four quadrants over the heterostructure 29], the potentials at the corners of the QD can be individually regulated. In Fig. 5 a we show the ground-state charge-density obtained from the exact diagonalization of a square QD [25], for device parameters placing it deep in the Wigner molecule regime. It can be seen that the charge-density is sharply peaked at four points, located close to the vertices of the QD. This structure arises from the Coulomb interaction between the electrons, which tends to force them apart into diagonally opposite corners of the dot. As there are two such diagonal states, degenerate in energy, we can understand the form of the ground-state by considering it to be essentially a superposition of these two states (with a small admixture of higher energy states). The four points at which the peaks occur define the sites on which the effective lattice-Hamiltonian operates, as shown in Fig. 5 b.

We take an effective lattice-Hamiltonian of the form:

$$
H=\sum_{\langle i, j\rangle, \sigma}\left[\tilde{t}\left(c_{i \sigma}^{\dagger} c_{j \sigma}+\text { H.c. }\right)+\tilde{V} n_{i} n_{j}\right]+\sum_{i}\left[\tilde{U} n_{i \uparrow} n_{i \downarrow}+E_{i}(t) n_{i}\right] .
$$

Here $\tilde{V}$ represents the Coulomb repulsion between electrons occupying neighboring sites, and $\tilde{U}$ is the standard Hubbard- $U$ term, giving the energy cost 
(a)

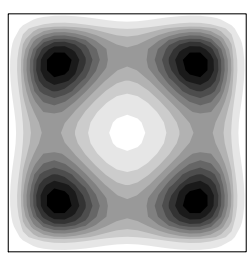

(b)

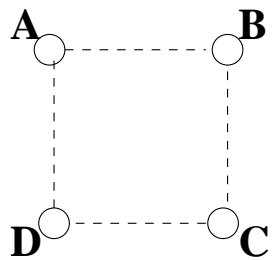

Fig. 5. (a) Ground-state charge-density for a two-electron square QD. GaAs material parameters are used, and the side-length of the QD is $800 \mathrm{~nm}$, placing it in the Wigner regime. The dark areas indicate peaks in the charge-density. (b) Lattice points used for the effective lattice-Hamiltonian.

for double-occupation of a site. As before, $E_{i}(t)$ denotes the electric potential at site $i$, which in general can have a static and a time-dependent component. In experiment, static offsets can arise either from deviations of the confining potential of the QD from the ideal geometry, or by the application of gating voltages to the corners of the QD. Applying corner potentials in this way would substantially enhance the stability of the Wigner molecule state, and could also be used to ensure that the multiplet of states included in the effective lattice-model is well-separated from the other excited states of the QD system. In this work, however, we do not explicitly consider the effects of static gates, and we neglect the influence of small, accidental offsets encountered in experiment as we expect them to have only minor effects, and indeed may even stabilize CDT [30. For convenience, we consider applying an $\mathrm{AC}$ field aligned with the $x$-axis of the $\mathrm{QD}$, which can be parameterized as:

$$
E_{A}=E_{D}=\frac{E}{2} \cos \omega t, \quad E_{B}=E_{C}=-\frac{E}{2} \cos \omega t
$$

where A,B,C,D label the sites as shown in Fig. 5 b. We emphasize that although we have the specific system of a semiconductor QD in mind, the effective-Hamiltonian we are using can describe a wide range of physical systems, including $2 \times 2$ arrays of connected QDs 31, and our results are thus of general applicability.

As for the case of the double QD, we include no spin-flip terms in (9) and so the singlet and triplet sectors are again decoupled. We choose to use initial states with singlet symmetry, which corresponds to the symmetry of the system's ground-state. Simple state counting reveals that the singlet sector has a dimension of ten, and can be spanned by the six states shown schematically in Fig.6, together with the four states in which each site is doubly-occupied. 


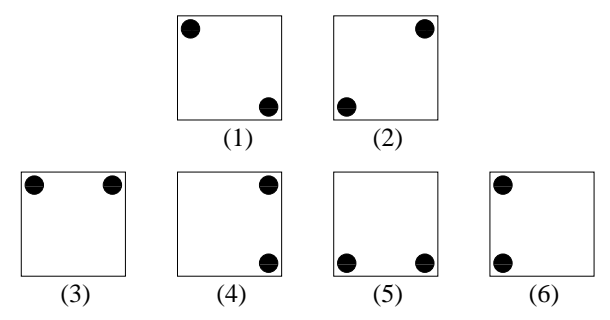

Fig. 6. Schematic representation of the two-particle basis states for the singlet sector of the Hamiltonian. The ground state of the QD is approximately a superposition of states (1) and (2).

\section{Results}

\subsection{Interacting electrons, double occupancy excluded}

We begin our investigation by taking the Hubbard- $U$ term to be infinitely large - that is, to work in the sub-space of states with no double occupation. Our Hilbert space is thus six-dimensional, and we use the states shown in Fig. 6 as a basis. We show in Fig.7 the time-dependent number occupation of the four sites at two different values of $E$, in both cases using state (6) as the initial state, and setting the AC frequency to $\omega=8$. In Fig.7la $E$ has a value of 100.0, and it can be clearly seen that the electrons perform driven Rabi oscillations between the left side of the QD and the right. Accordingly, the occupation number of the sites varies continuously between zero and one. In Fig. $7 \mathrm{~b}$, however, we see that changing the electric potential to a value of $E=115.7$ produces dramatically different behavior. The occupations of sites A and D only vary slightly from unity, while sites B and C remain essentially empty throughout the time-evolution. Only a small amount of charge can transfer per period of the driving field between the left and right sides of the system, producing the small spikes visible in this figure. The amplitude of these features is extremely small, however, indicating that the tunneling between left and right sides has been almost totally destroyed.

To confirm that CDT is occurring, we present in Fig.8 a comparison of the amplitude of the oscillations of $n_{A}$ with the quasi-energy spectrum, as a function of the electric potential $E$. Similarly to the double QD system, we can see in Fig.8 8a that the quasi-energies have two different regimes of behavior. The first of these is the weak-field regime, $E<\tilde{V}$, at which the driving field does not dominate the dynamics. In this regime the quasi-energy spectrum, and correspondingly, the amplitude of oscillations shows little structure. The second regime occurs at strong values of potential, $E>\tilde{V}$, for which the quasi-energy spectrum clearly shows a sequence of close approaches. In Fig. \&gc we show an enlargement of one of these approaches which reveals it to be an avoided crossing. Employing the perturbative method described in Section 2.2 demonstrates that the two quasi-energies involved in these avoided crossings 


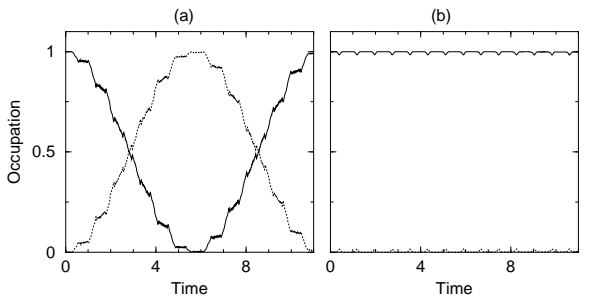

Fig. 7. Time development of the system for $\tilde{U}$ infinite, $\tilde{V}=80$ and $\omega=8$ : (a) electric potential, $\mathrm{E}=100.0$ (b) $\mathrm{E}=115.7$. Solid line indicates the occupation of sites $\mathrm{A}$ and $\mathrm{D}$, the dotted line the occupation of sites $\mathrm{B}$ and $\mathrm{C}$.

are described by $\pm 2 J_{n}(E / \omega)$, where $n$ is equal to $\tilde{V} / \omega$. We may thus again think of $n$ as signifying the number of photons the system needs to absorb to overcome the Coulomb repulsion between the electrons occupying neighboring sites. The results in Fig.8b and Fig.8d clearly show that the locations of the avoided crossings correspond exactly to quenching of the oscillations in $n_{A}$, and so confirm that CDT indeed occurs at these points.

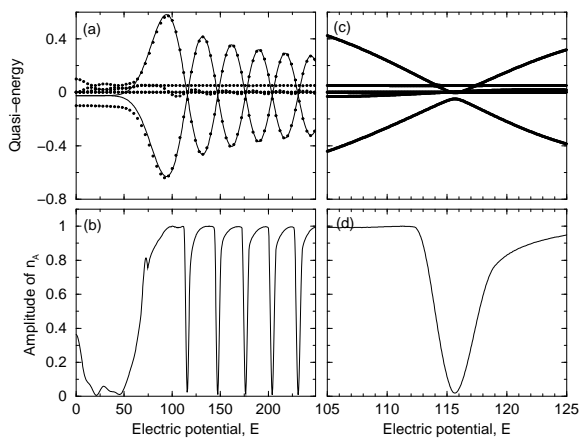

Fig. 8. (a) Quasi-energies of the system for $\tilde{U}$ infinite, $\tilde{V}=80$ and $\omega=8$ : circles $=$ exact results, lines $=$ perturbative solution $\left[ \pm 2 J_{10}(E / \omega)\right]$. (b) Amplitude of oscillation of the occupation of site A. (c) Detail of quasi-energy spectrum, showing an avoided crossing. (d) Detail of amplitude of oscillations.

\subsection{Interacting electrons, double-occupancy permitted}

We now take the most general case, and consider the competition between the $\tilde{U}$ and $\tilde{V}$ terms. Setting $\tilde{U}$ to a finite value means that the four doublyoccupied basis states are no longer energetically excluded from the dynamics, and accordingly we must take the full ten-dimensional basis set. 
Although it is difficult to obtain precise estimates for the values of parameters of the effective Hamiltonian, it is clear that in general $\tilde{U}>\tilde{V}$. Accordingly we choose the parameters $\tilde{U}=160, \tilde{V}=16$ to separate the two energy-scales widely for our investigation. We again set the frequency of the $\mathrm{AC}$ field to $\omega=8$, and in Fig. $9 \mathrm{a}$ we show the quasi-energy spectrum obtained by sweeping over the field strength. It is immediately clear from this figure that for electric potentials $E<\tilde{U}$ the form of the spectrum is extremely similar to the infinite- $\tilde{U}$ case. Performing perturbation theory confirms that, as in the previous case, the behavior of the quasi-energies is given by $\pm 2 J_{n}(E / \omega)$ where $n=\tilde{V} / \omega$. We show in Fig. $9 \mathrm{~b}$ the amplitude of the oscillations of $n_{A}$ when the system is initialized in state (6), which demonstrates that at the locations of the avoided crossings the tunneling parallel to the field is again quenched.

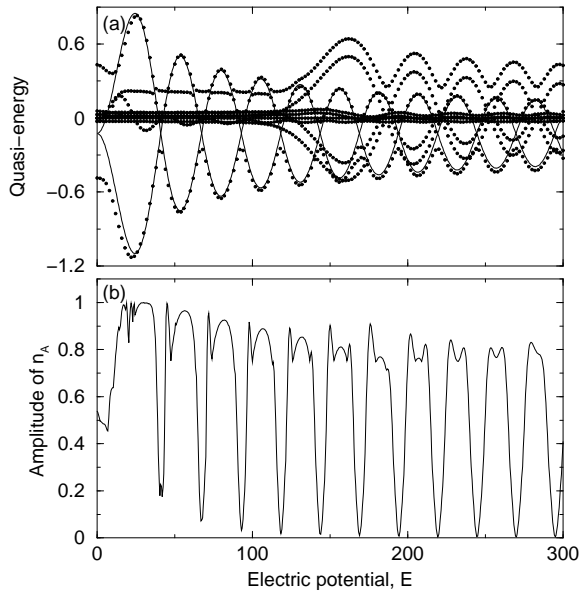

Fig. 9. (a) Quasi-energies of the system for $\tilde{U}=160$ and $\tilde{V}=16, \omega=8$ : circles $=$ exact results, lines=perturbative solution $\left[ \pm 2 J_{2}(E / \omega)\right]$. (b) Amplitude of oscillation of the occupation of site A, with (6) as the initial state.

When the electric potential exceeds the value of $\tilde{U}$, however, new structure appears in the quasi-energy spectrum. A group of four quasi-energies, that for weaker fields cluster around zero, become "excited" and make a sequence of avoided crossings as the field strength is increased. Perturbation theory predicts that these high-field quasi-energies are given by $\pm 2 J_{m}(E / \omega)$, where $m=(\tilde{U}-\tilde{V}) / \omega$, and thus these avoided crossings arise when the absorption of $m$ photons equates to the electrostatic energy difference between the two electrons being on neighboring sites, and doubly-occupying one site. This then indicates that this structure arises from the coupling of the AC field to the doubly-occupied states. 
To probe this phenomenon, we time-evolve the system from an initial state consisting of two electrons occupying site A. In Fig.10b it can be seen that for electric potentials weaker than $\tilde{U}$ the amplitude of the oscillations in $n_{A}$ remains small, and shows little dependence on the field. As the potential exceeds $\tilde{U}$, this picture changes, and the AC field drives large oscillations in $n_{A}$, and in fact mainly forces charge to oscillate between sites $\mathrm{A}$ and B. At the high-field avoided crossings, however, the tunneling between A and B is suppressed, which shuts down this process. Instead, the only timeevolution that the system can perform consists of undriven Rabi oscillations between sites $\mathrm{A}$ and $\mathrm{D}$, perpendicular to the field. As these oscillations are undriven they have a much longer time-scale than the forced dynamics, and thus during the interval over which we evolve the system the occupation of A only changes by a small amount, producing the very sharp minima visible in Fig $10 \mathrm{~b}$, centered on the roots of $J_{m}(E / \omega)$.

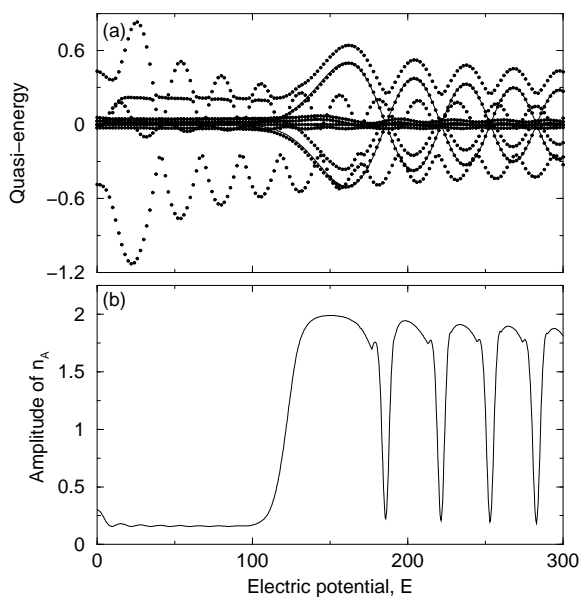

Fig. 10. (a) Quasi-energies of the system for $\tilde{U}=160$ and $\tilde{V}=16, \omega=8$ : circles $=$ exact results, lines $=$ perturbative solution $\left[ \pm 2 J_{18}(E / \omega)\right]$. (b) Amplitude of oscillation of the occupation of site A, with site A doubly-occupied as the initial state.

As the tunneling perpendicular to the field is undriven, it is straightforward to evaluate the time evolution of the initial state, if we assume that the left side of the QD is completely decoupled from the right side. The occupation of sites $\mathrm{A}$ and $\mathrm{D}$ is then given by:

$$
n_{A}(t)=1+\cos \Omega_{R} t, \quad n_{D}=1-\cos \Omega_{R} t
$$

where $\Omega_{R}=4 \tilde{t}^{2} /(\tilde{U}-\tilde{V})$. In Fig. 11 we display the occupations of sites A and $\mathrm{D}$ as a function of time, for two values of electric potential. At the first value, 
$E=200$, tunneling between the left and right sides of the QD is not quenched, and accordingly the occupation of the two sites varies rapidly between zero and two as the electrons are driven by the AC field around the system. The second value, $E=185.8$, corresponds to the first high-field avoided crossing. It can be clearly seen that the charge oscillates between sites A and B, with a frequency of $\Omega_{R}$. These Rabi oscillations are damped, however, indicating that the isolation between the left and right sides of the QD is not perfect. In this sense we can regard the two sites $B$ and $C$ as providing an environment, causing the quantum system composed of sites A and D to slowly decohere in time. When the tunneling between the left and right sides of the QD is strong, for example at $E=200$, this decoherence occurs very rapidly. By moving to an avoided crossing, however, and suppressing the tunneling, the rate of mixing between the two sides of the QD can be considerably reduced, and is just limited by the separation in energy between the two quasi-energies. Tuning the parameters of the driving field therefore gives us a simple and controllable way to investigate how a two-electron wavefunction can decohere in a QD.
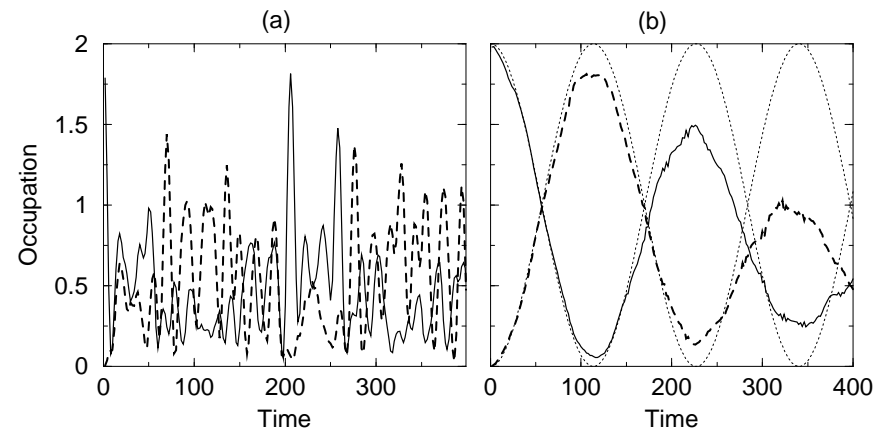

Fig. 11. Time development of the $\tilde{U}=160$ and $\tilde{V}=16$ system for $\omega=8$ : (a) electric potential, $\mathrm{E}=200.0$ (no CDT) (b) $\mathrm{E}=185.8$ (CDT). Thick solid line indicates the occupation of site A, the thick dotted line the occupation of site D. Dotted lines in (b) show the Rabi oscillations of the isolated two-site system, Eq.11.

\section{Conclusions}

In this work we have studied the interplay between Coulomb interactions and an AC driving field in two different configurations of QDs. For the case of the double QD system we have found that the system presents two different types of behavior, depending on whether the driving field dominates the interaction energy. For weak driving, we have found the surprising result that a suitable 
AC field can nonetheless freeze the time-evolution of a doubly-occupied QD despite the Coulomb interaction, and that this can be understood by means of an effective two-level model. At high field strengths the system is only driven strongly by the field at frequencies for which an integer number of quanta, $n$, is equal to the interaction energy. When this condition is satisfied CDT is again able to occur at certain well-defined parameters of the field, and by using Floquet theory we have shown that these points correspond to the roots of $J_{n}(E / \omega)$.

Strong electronic correlations allowed us to use an effective lattice model of just four sites to treat the square QD, by taking advantage of the natural discretization of the system in a Wigner molecule state. In the effective model, the inter-electron Coulomb interaction is described by two parameters, $\tilde{U}$ and $\tilde{V}$, and the dynamics of the system consists essentially of tunneling from corner to corner, along the perimeter of the QD. We find again that when the frequency of the driving field is in resonance with the Coulomb gap (that is, $m \omega=\tilde{V}$ or $m \omega=(\tilde{U}-\tilde{V}))$ charge is able to circulate freely around the system, except at sharply defined field strengths at which tunneling parallel to the field is destroyed. Floquet theory again proved an excellent tool to understand this behavior, and revealed that these points correspond to the roots of $J_{m}(E / \omega)$.

We have thus shown that AC-fields may not only be used as a spectroscopic tools to probe the electronic structure of QD systems, but can also be used to dynamically control the time-evolution of the system. Possible applications of CDT range from stabilising the leakage of trapped electrons in physical realisations of quantum bits, to acting as "electron tweezers" by destroying or restoring tunneling between regions of a mesoscopic device. The tunability of the CDT effect, and its ability to discriminate between doublyoccupied and singly-occupied states, make it an excellent means for rapid manipulation of the dynamics of strongly correlated electrons in mesoscopic systems.

\section{Acknowledgments}

This work was supported by the Spanish DGES grant MAT2002-02465, by the European Union TMR contract FMRX-CT98-0180 and by the European Community's Human Potential Programme under contract HPRN-CT-200000144, Nanoscale Dynamics.

\section{References}

1. C.E. Creffield and G. Platero, Phys. Rev. B 65, 113304 (2002).

2. M. Grifoni and P. Hänggi, Phys. Rep. 304, 229 (1998).

3. C.E. Creffield and G. Platero, Phys. Rev. B 66, 2353XX (2002). 
4. M.A. Kastner, Phys. Today 46, 24 (1993); R.C. Ashoori, Nature (London) 379, 413 (1996).

5. R.H. Blick, D. Pfannkuche, R.J. Haug, K. von Klitzing and K. Eberl, Phys. Rev. Lett. 80, 4032 (1998).

6. T.H. Oosterkamp, T. Fujisawa, W.G. van der Wiel, K. Ishibashi, R.V. Hijman, S. Tarucha and L.P. Kouwenhoven, Nature (London) 395, 873 (1998).

7. P.W. Anderson, Phys. Rev. 109, 1492 (1958).

8. F. Grossmann, T. Dittrich, P. Jung and P. Hänggi, Phys. Rev. Lett. 67, 516 (1991).

9. J.H. Shirley, Phys. Rev. 138, B979 (1965).

10. M. Holthaus, Z. Phys. B 89, 251 (1992).

11. F. Grossmann and P. Hänggi, Europhys. Lett. 18, 571 (1992).

12. K. Jauregui, W. Häusler and B. Kramer, Erophys. Lett. 24, 581 (1993).

13. B.E. Cole, J.B. Williams, B.T. King, M.S. Sherwin and C.R. Stanley, Nature (London) 410, 60 (2001); D. Vion, A. Aasime, A. Cottet, P. Joyez, H. Pothier, C. Urbina, D. Esteve and M.H. Devoret, Science 296, 886 (2002).

14. P.I. Tamborenea and H. Metiu, Phys. Rev. Lett. 83, 3912 (1999).

15. C.H. Bennett and D.P. DiVincenzo, Nature (London) 404, 247 (2000).

16. J.H. Jefferson and W. Häusler, Phys. Rev. B 54, 4936 (1996).

17. J. von Neumann and E.P. Wigner, Phys. Z 30, 467 (1929).

18. H. Sambe, Phys. Rev. A 7, 2203 (1973).

19. T. Fujisawa, D.G. Austing, Y. Tokura, Y. Hirayama and S. Tarucha, Phys. Rev. Lett. 88, 236802 (2002).

20. P. Zhang and X.-G. Zhao, Phys. Lett. A 271, 419 (2000).

21. E.P. Wigner, Phys. Rev. 46, 1002 (1934).

22. B. Tanatar and D.M. Ceperley, Phys. Rev. B 39, 5005 (1989).

23. S. Akbar and I.-H. Lee, Phys. Rev. B 63, 165301 (2001).

24. P.A. Schulz, P.H. Rivera, and N. Studart, Phys. Rev. B 66, 195310 (2002).

25. C.E. Creffield, W. Häusler, J.H. Jefferson and S. Sarkar, Phys. Rev. B 59, 10719 (1999).

26. C.E. Creffield, J.H. Jefferson, S. Sarkar and D.L.J. Tipton, Phys. Rev. B 62, 7249 (2000).

27. W. Häusler, Physica B 222, 43 (1996).

28. M. Koskinen, M. Manninen, B. Mottelson and S.M. Reimann, Phys. Rev. B 63, 205323 (2001).

29. D.G. Austing, T. Honda and S. Tarucha, Semicond. Sci. Technol. 12, 631 (1997).

30. J.T. Stockburger, Phys. Rev. E 59, R4709 (1999).

31. C.A. Stafford and S. Das Sarma, Phys. Rev. Lett. 72, 3590 (1994); R. Kotlyar and S. Das Sarma, Phys. Rev. B 55, R10205, (1997). 